\title{
A new approach for investigating the relative contribution of basal glucose and postprandial glucose to $\mathrm{HbA1}_{\mathrm{C}}$
}

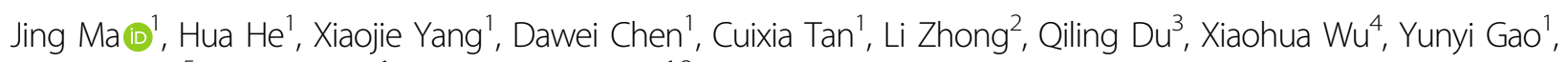
Guanjian Liư ${ }^{5}$ Chun Wang ${ }^{1}$ and Xingwu Ran (1),3

\begin{abstract}
To develop an accurate method for evaluating the relative contributions of basal glucose (BG) and postprandial glucose (PPG) to glycated haemoglobin ( $\mathrm{HbA1c}$ ) in subjects with hyperglycaemia using a Continuous Glucose Monitoring System (CGMS ${ }^{\circledR}$ ). The subjects were divided into the normal glucose tolerance (NGT), impaired glucose tolerance (IGT), newly-diagnosed type 2 diabetes (NDDM), and drug-treated type 2 diabetes (T2DM) groups. We evaluated the relative contributions of $\mathrm{BG}$ and PPG to $\mathrm{HbA1c}$ in patients with hyperglycaemia according to three different baseline values. Subjects $(n=490)$ were grouped as follows: 92 NGT, 36 IGT, 131 NDDM, and 231 T2DM. The relative contributions of PPG to HbA1c were calculated using baseline values of $6.1 \mathrm{mmol} / \mathrm{L}, 5.6 \mathrm{mmol} / \mathrm{L}$, and the 24-h glucose curve of the NGT group. The relative contribution of PPG to HbA1c decreased progressively from the IGT group to the T2DM group. Compared with the 24-h glucose curve as the baseline, the relative contribution of PPG was overestimated in $9.04 \%$ and $1.76 \%$ of the subjects when $6.1 \mathrm{mmol} / \mathrm{L}$ and $5.6 \mathrm{mmol} / \mathrm{L}$ were used as baselines, respectively $(P<0.01)$, in T2DM patients. The 24-h glucose curve of NGT is more suitable for studying the relative contributions of BG and PPG to HbA1c and it is more precise, as it considers physiological fluctuations in NGT after meals. However, $5.6 \mathrm{mmol} / \mathrm{L}$ can be used when the $24-\mathrm{h}$ glucose curve for NGT is unavailable; using $6.1 \mathrm{mmol} / \mathrm{L}$ as a baseline value may overestimate the contribution to the $\mathrm{HbA1c}$. There is no unified standard for assessing the contributions of basal glucose (BG) and postprandial glucose (PPG) to HbA1c. The 24-h glucose curve of NGT is more suitable for studying the relative contributions of $\mathrm{BG}$ and PPG to HbA1c, as it considers physiological fluctuations in NGT after meals. However, $5.6 \mathrm{mmol} / \mathrm{L}$ can be used when the 24-h glucose curve for NGT is unavailable; using $6.1 \mathrm{mmol} / \mathrm{L}$ as a baseline value may overestimate the contribution to the HbA1c.
\end{abstract}

\section{Introduction}

Glycaemic control is a cornerstone in reducing the morbidity and mortality of diabetes. Previous studies have focused on haemoglobin A1c (HbA1c) and fasting plasma glucose (FPG)/basal glucose (BG) values to determine the

\footnotetext{
Correspondence: Xingwu Ran (ranxingwu@163.com)

'Innovation Center for Wound Repair, Diabetic Foot Care Center, Department of Endocrinology and Metabolism, West China Hospital, Sichuan University,

Chengdu, China

2Department of Endocrinology and Metabolism, The First People's Hospital of Longquanyi District, Chengdu, China

Full list of author information is available at the end of the article

These authors contributed equally: Jing Ma, Hua He
}

level of glycaemic control. Studies have demonstrated that only comprehensive control of HbA1c, BG, and postprandial glucose (PPG) can prevent the occurrence and progression of vascular complications ${ }^{1}$. The United Kingdom Prospective Diabetes Study showed that FPG and HbA1c are the main risk factors for cardiovascular complications ${ }^{2}$, and reduced FPG levels are associated with reduced cardiovascular mortality in diabetes ${ }^{3}$. However, other studies showed that PPG is associated with cardiovascular events and all-cause mortality ${ }^{4,5}$.

In 2003, Monnier et al. ${ }^{6}$ first proposed that FPG and PPG contribute to HbA1c in patients with type 2 diabetes

\section{(c) The Author(s) 2021}

(c) (i) Open Access This article is licensed under a Creative Commons Attribution 4.0 International License, which permits use, sharing, adaptation, distribution and reproduction cc) in any medium or format, as long as you give appropriate credit to the original author(s) and the source, provide a link to the Creative Commons license, and indicate if changes were made. The images or other third party material in this article are included in the article's Creative Commons license, unless indicated otherwise in a credit line to the material. If material is not included in the article's Creative Commons license and your intended use is not permitted by statutory regulation or exceeds the permitted use, you will need to obtain permission directly from the copyright holder. To view a copy of this license, visit http://creativecommons.org/licenses/by/4.0/. 
mellitus (T2DM) being administered antidiabetic treatment except for insulin and acarbose. Since the first milestone article was published, an increasing number of researchers had addressed these relationships. However, the studies were conducted using various methods and yielded different results. Riddle et al. ${ }^{7}$ analysed data from six randomised controlled trials and found that the relative contribution of BG played a major role $(76-80 \%)$ when the $\mathrm{HbA1c}$ was $>7.0 \%$. However, the $\mathrm{A} 1_{\mathrm{C}}$-derived average glucose study ${ }^{8}$ showed that BG was not a clear indicator of general glycaemia. Table 1 summarizes 17 relevant studies ${ }^{6,7,9-25}$ published up to Jan 1, 2021. It shows the complex results on the relative contribution of PPG to HbA1c in diverse subjects, treatment regimens, and baseline criterias. Eleven articles used $5.5 / 5.6 \mathrm{mmol} / \mathrm{L}$ as baseline value. The value of $5.6 \mathrm{mmol} / \mathrm{L}(100 \mathrm{mg} / \mathrm{dL})$ was chosen to align with the recommendation of the American Diabetes Association for the upper limit of normal fasting glucose levels ${ }^{26}$. Four articles utilized $6.1 \mathrm{mmol} / \mathrm{L}(110 \mathrm{mg} / \mathrm{dL})$ as a baseline value, which has been described as the upper limit of normal fasting glucose levels according to the World Health Organization ${ }^{27}$. Two articles only discussed the correlation between PPG and HbA1c, without the specific contribution of each factor $^{10,12}$. Moreover, one study firstly used the 24-h glucose profiles of the NGT curve as a baseline value, firstly pointed and discussed the influence of different baseline values, and found that the relative contribution of PPG may overestimated by approximately $10-20 \%$ when $6.1 \mathrm{mmol} / \mathrm{L}$ was used as a baseline, compared with the 24$h$ glucose profiles of the NGT curve ${ }^{17}$. Bergenstal ${ }^{28}$ stated that CGM has transformed glucose control and can be used to identify glucose excursions in patients with diabetes. The recent development of some new therapies specifically aimed at reducing BG or PPG has further increased the interest for studying the complex relationship between the contributions of BG and PPG, yet have yielded conflicting results ${ }^{20,21,23,25}$. Recently, the results of a randomised crossover trial conducted by CGMS pointed that the conflicting results may be related to differences in the study population, methodologies, etc ${ }^{29}$. Therefore, this study was conducted to develop a more accurate method for evaluating the relative contributions of BG and PPG to HbA1c by using a CGM.

\section{Materials and methods}

\section{Population and subject selection}

Subjects with a normal glucose tolerance (NGT), impaired glucose tolerance (IGT), newly diagnosed type 2 diabetes (NDDM), and drug-treated type 2 diabetes (T2DM) from multiple communities including the Chenghua, Xindu, and Longquanyi districts in Chengdu city and West China Hospital of Sichuan University were consecutively enrolled in this study. The study was approved by the Biomedical Research Ethics Committee of West China Hospital of Sichuan University. All participants provided written informed consent.

All participants were 18-75 years of age. Participants in the NGT group were in good health without obesity, hyperlipidaemia, diabetes, or hypertension. Normal blood pressure was defined as $90-139 / 60-89 \mathrm{mmHg}$, and normal lipid profiles included triglyceride $<2.22 \mathrm{mmol} / \mathrm{L}$, total cholesterol $<6.22 \mathrm{mmol} / \mathrm{L}$. The body mass indices were $18.5-24.9 \mathrm{~kg} / \mathrm{m}^{2}$. Drug-treated patients met the diagnostic criteria for T2DM according to the World Health Organization ${ }^{27}$ standard over 6 months and were treated with fixed oral anti-diabetic drugs for at least 3 months before the study. Participants who had impaired liver (aspartate aminotransferase or alanine aminotransferase levels 2-fold the upper the limit of the normal range) or renal (serum creatinine 1.2-fold the upper normal range or estimated glomerular filtration rate $<30 \mathrm{~mL} / \mathrm{min}$ ) function or had other comorbidities or conditions that may lead to severe blood glucose fluctuation, such as malignant tumours, human immunodeficiency virus infection, acute infections, trauma, or surgery were excluded from the study. Insulin, DPP-4 inhibitors, GLP-1 receptor agonists, and acarbose treatments were also excluded to avoid any bias in the interpretation of the contribution of PPG increments to overall hyperglycaemia, as they exert specific effects on PPG excursions.

\section{Outcome measures}

Medical history data and anthropometric data (blood pressure, height, weight, etc.) were recorded. An oral glucose tolerance test(OGTT) was performed for each participant. The plasma glucose concentration was measured by the glucose oxidase method. HbA1c was measured by high-performance liquid chromatography (BioRad-10, Hercules, CA, USA). Biochemical parameters, including triglyceride, total cholesterol, high-density lipoprotein-cholesterol, low-density lipoprotein-cholesterol, liver function, and renal function were evaluated with an automatic biochemical analyser (D/P/ISE, Roche, Basel, Switzerland).

\section{Formulation of mixed-meals}

All subjects received nutritional assessments and dietary instructions from the nutritionist and endocrinologist of our hospital based on the China Medical Nutritional Therapy Guideline for Diabetes $(2013)^{30}$. Adjusted the individualized energy standard mainly according to the subject's BMI, age, activity level, and then individualized dietary recipes plan was formulated. The ratio of carbohydrate, proteins, and fats were $45-60 \%, 15-20 \%$, and $25-35 \%$. For overweight or obese subjects, the ratio of fat was controlled within 30\% (Supplementary Table 1). 


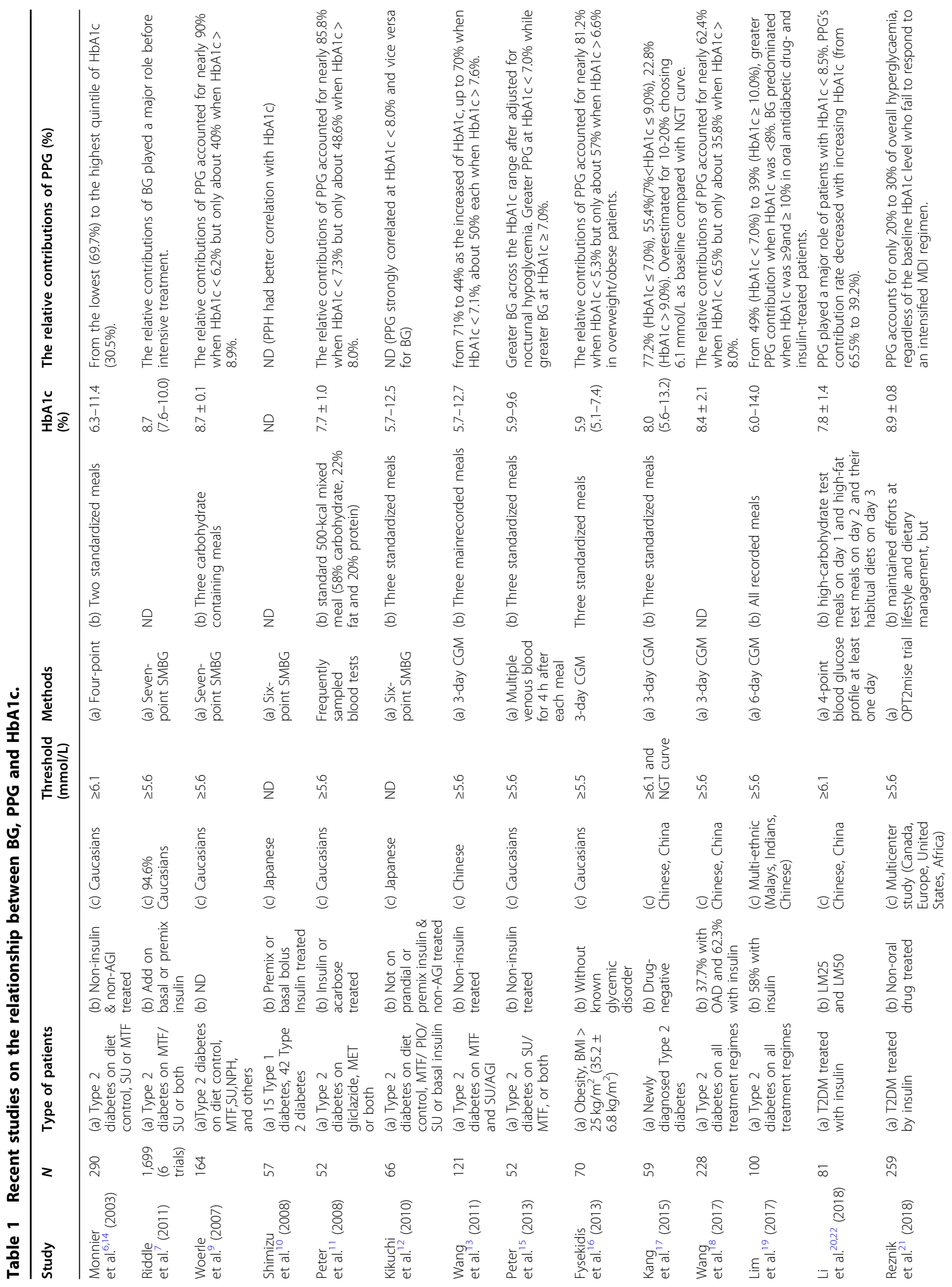




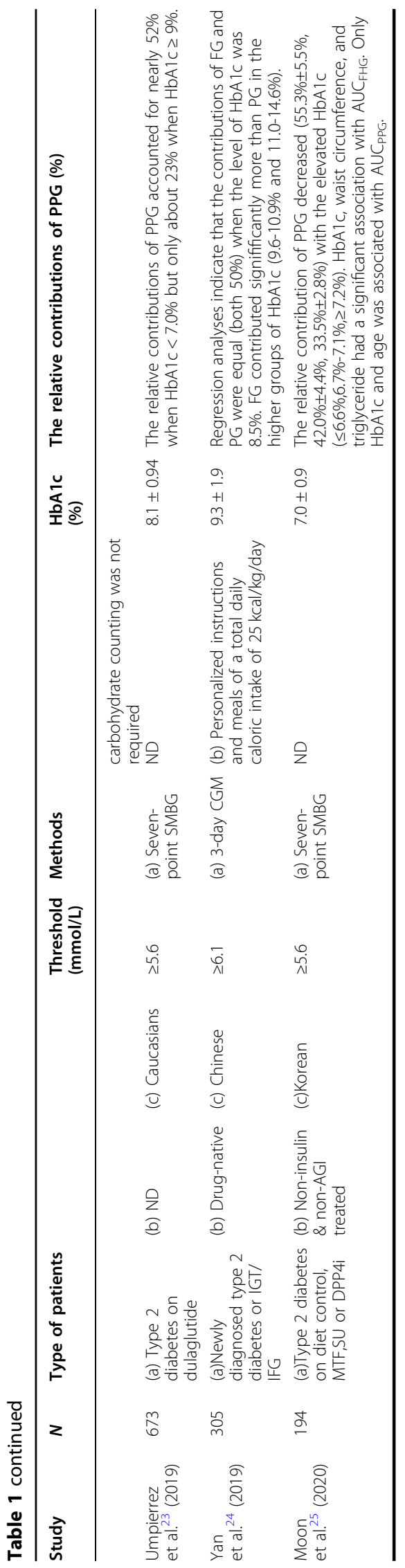

Subjects were instructed to have breakfast, lunch, and dinner at 7:00-8:00AM, 11:00-12:00 AM, and 5:00-6:00 PM, respectively. Subjects were not permitted to eat between meals and were required to record the mealtime, amount, cooking style and species of each meal carefully. In this study, we adopted a relatively fixed-time, fixedratio diet to avoid bias caused by differences in diet. All participants refrained from consuming alcohol, strong tea, and coffee during the monitoring period ${ }^{30,31}$.

\section{Installation of CGMS}

CGMS ${ }^{\circledR}$ Gold (Medtronic MiniMed, Northridge, CA, USA) was administered for CGM in this study. The glucose sensor was inserted into the subcutaneous tissue of the abdomen of all participants at 08:00-09:00 in the morning to monitor the glucose levels in the interstitial fluid for at least $72 \mathrm{~h}$. Finger stick blood glucose levels were checked to calibrate and four calibrations per day (before meals and at bedtime) were conducted with an Accu-Chek Integra Blood Glucose Meter (Roche) during the CGM period. The subjects returned to our hospital to download the data from CGMS after 3 days. The data analysed using the Solution Software v3.0c.

\section{Calculation method and three baseline criteria}

The overall additional hyperglycemia, which can be further divided into two subcomponents: the basal glucose (BG) and postprandial glucose (PPG). One question to be raised is to define the word "Basal glucose", with the word "basal" being probably more appropriate than "fasting". As soon as these two components of hyperglycemia were identified, diabetologists have paid increasing attention to their respective contributions to the overall hyperglycemia in hyperglycemia subjects. BG is the component that remains after the PPG has been subtracted from the overall hyperglycemia. Three baseline criterias, which were $6.1 \mathrm{mmol} / \mathrm{L}$, the upper limit of fasting blood glucose in normal subjects according to the World Health Organization standard $^{27}$ in method A; $5.6 \mathrm{mmol} / \mathrm{L}$, the upper limit of fasting blood glucose in normal subjects according to the American Diabetes Association standard ${ }^{26}$ in method B and 24-h glucose profiles of the NGT curve as baseline in method $C$, respectively, were performed to evaluate the contribution of PPG to HbA1c. The area under the curve and above the baseline criteria represented excessive hyperglycaemia, named as $\mathrm{AUC}_{-\mathrm{T}}$. This value was calculated as $\mathrm{AUC}_{-\mathrm{T}}=\mathrm{AUC}_{\mathrm{T} 2 \mathrm{DM}}-\mathrm{AUC}_{\text {baseline }}$ when the glucose profile of subjects was always higher than that of the baseline value, as condition A (Fig. 1). However, if the glucose profile was under the baseline, as condition B (Fig. 2), AUC - $_{\text {T }}$ was only calculated using glucose values above the baseline criteria, and the area below the baseline point was not included in the calculation of overall hyperglycaemia. AUC-PPG reflects the 4-h 


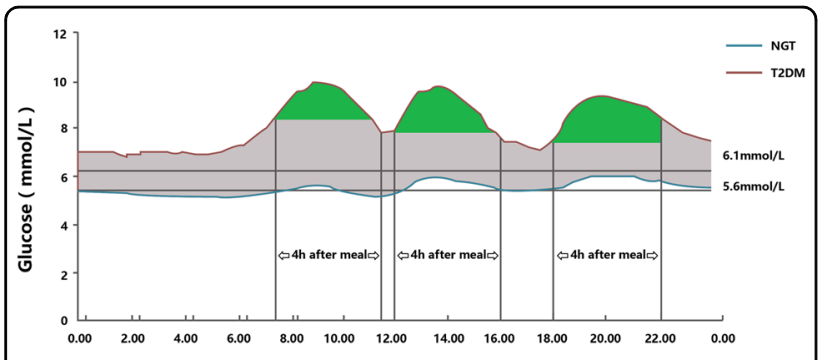

Fig. 1 Calculationused for the AUC under condition A. The green part represents AUC-pPG. The gray partrepresents AUC-BG (AUC-T - AUCPPG).

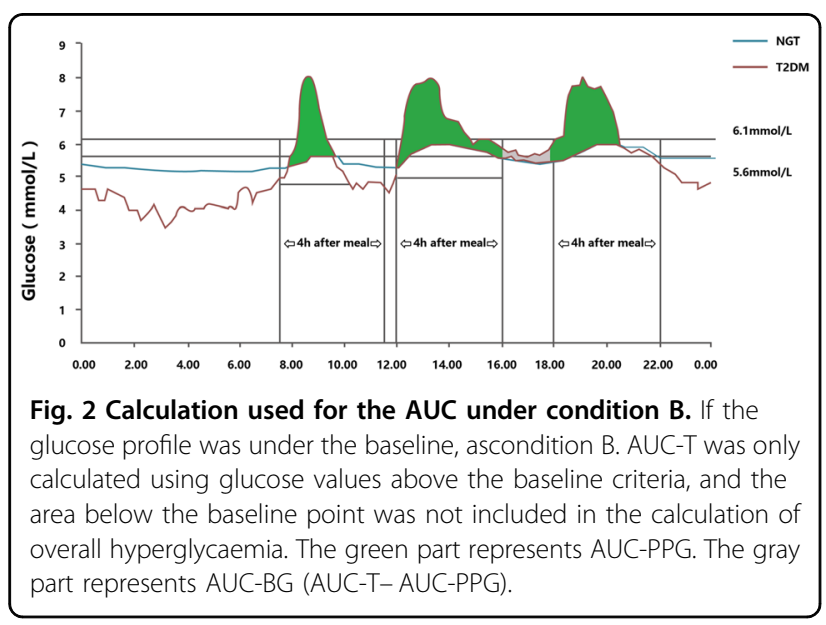

glucose incremental area above the pre-prandial glucose values assessed just before starting breakfast, lunch, and dinner, and $\mathrm{AUC}_{-\mathrm{PPG}-\mathrm{Total}}=\mathrm{AUC}_{\text {-PPG-b }}+\mathrm{AUC}_{-\mathrm{PPG}-\mathrm{I}}+$ AUC-PPG-d. The contribution of PPG to overall hyperglycaemia was calculated as AUC $_{\text {-PPG-Total }} / \mathrm{AUC}_{-\mathrm{T}} \times 100 \%$. The contribution of $\mathrm{BG}$ to overall hyperglycaemia was calculated as $\left(\mathrm{AUC}_{-\mathrm{T}}-\mathrm{AUC}_{\text {-PPG-Total }}\right) / \mathrm{AUC}_{-\mathrm{T}} \times 100 \%$.

\section{Statistical analysis}

Statistical analyses were performed using SPSS 21.0 software (SPSS, Inc., Chicago, IL, USA). Data were expressed as the means \pm SDs or median with the quartile range. One-way analysis of variance was used among the studied groups to test differences in normally distributed data. The contributions of PPG under the three different baseline criteria were analysed using the Wilcoxon signed rank test between groups. All tests were 2 -sided, and $p$-values below 0.05 were considered as statistically significant.

\section{Results}

\section{Clinical and laboratory data}

A total of 490 subjects were enrolled in this study, including 92 NGT, 36 IGT, 131 NDDM, and 231 T2DM subjects. Table 2 shows the clinical and laboratory data of all subjects. The average age was $53.03 \pm 12.00$ years
(21-77 years) and average $\mathrm{HbA1c}$ value was $6.96 \pm 1.26 \%$ (4.5-12.7\%). The blood lipid parameters were in the normal reference value range, however, from the IGT group to the NDDM group, age, body mass index, triglyceride, total cholesterol, low-density lipoprotein-cholesterol were also gradually increased $(p<0.05)$. The NDDM group showed higher levels of systolic blood pressure, diastolic blood pressure, HbA1c, triglyceride, total cholesterol, low-density lipoprotein-cholesterol parameters, and a lower level of high-density lipoproteincholesterol compared to those in the T2DM group after drug treatment.

\section{Relative contribution of PPG to HbA1c calculated by three baseline criteria}

The relative contributions of PPG according to the three baseline criteria were significant with each other among all participants $(p<0.01)$. As shown in Table 3 , the relative contributions of PPG to HbA1c calculated by methods A, $\mathrm{B}$, and $\mathrm{C}$ for IGT were $80.85 \%, 72.36 \%$, and $67.68 \%$; for NDDM were $48.76 \%, 39.63 \%$, and $37.85 \%$; for T2DM were $39.46 \%, 33.22 \%$, and 31.47\%; and for NDDM + T2DM were $42.82 \%, 35.54 \%$, and $33.78 \%$, respectively. Based on these results, the NGT curve reflects physiological fluctuations in normal people after each meal, which is more accurate as a baseline criterion. In all subjects with hyperglycaemia, the relative contributions of PPG decreased progressively from the IGT group to the T2DM group regardless of the baseline criteria used, whereas the relative contributions of BG increased gradually (Fig. 3). Further analysis showed that the relative contribution of PPG was overestimated by approximately $13.16 \%$ when $6.1 \mathrm{mmol} / \mathrm{L}$ was used as a baseline, compared to when the NGT curve was used as the baseline in the IGT group. It was also overestimated in NDDM and T2DM groups $(p<$ 0.01 ) (Table 3). We also found that from the T2DM group to the IGT group, the overestimated level of the relative contribution of PPG to HbA1c also gradually increased (Fig. 4). Compared with method $\mathrm{C}$, the relative contribution of PPG was overestimated by $9.04 \%$ and $1.76 \%$ when $6.1 \mathrm{mmol} / \mathrm{L}$ and $5.6 \mathrm{mmol} / \mathrm{L}$ were used as a baseline, respectively, for patients with T2DM.

\section{Discussion}

More and more studies focused on the relative contributions of BG and PPG to HbA1c, but different studies use different baseline criteria. Regardless of the previous milestone articles, or the two newly published articles ${ }^{24,25}$, because of the different baseline values selected, quite different research results have been obtained. Yet there is not any research to explore the influence with different baseline criteria on the results. This is the first prospective study to evaluate the relative contributions of BG and PPG to HbA1c in different subjects with hyperglycaemia 


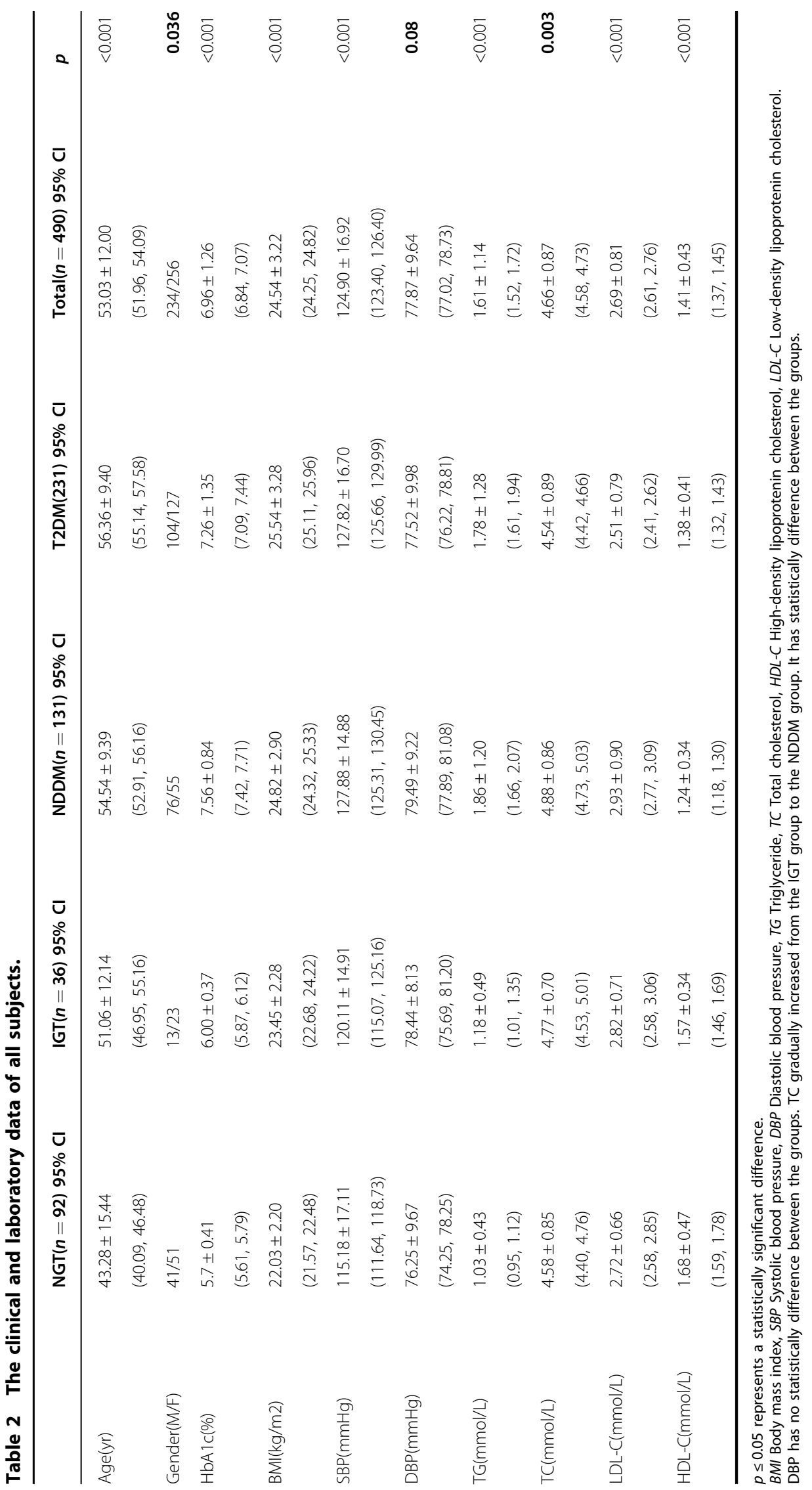




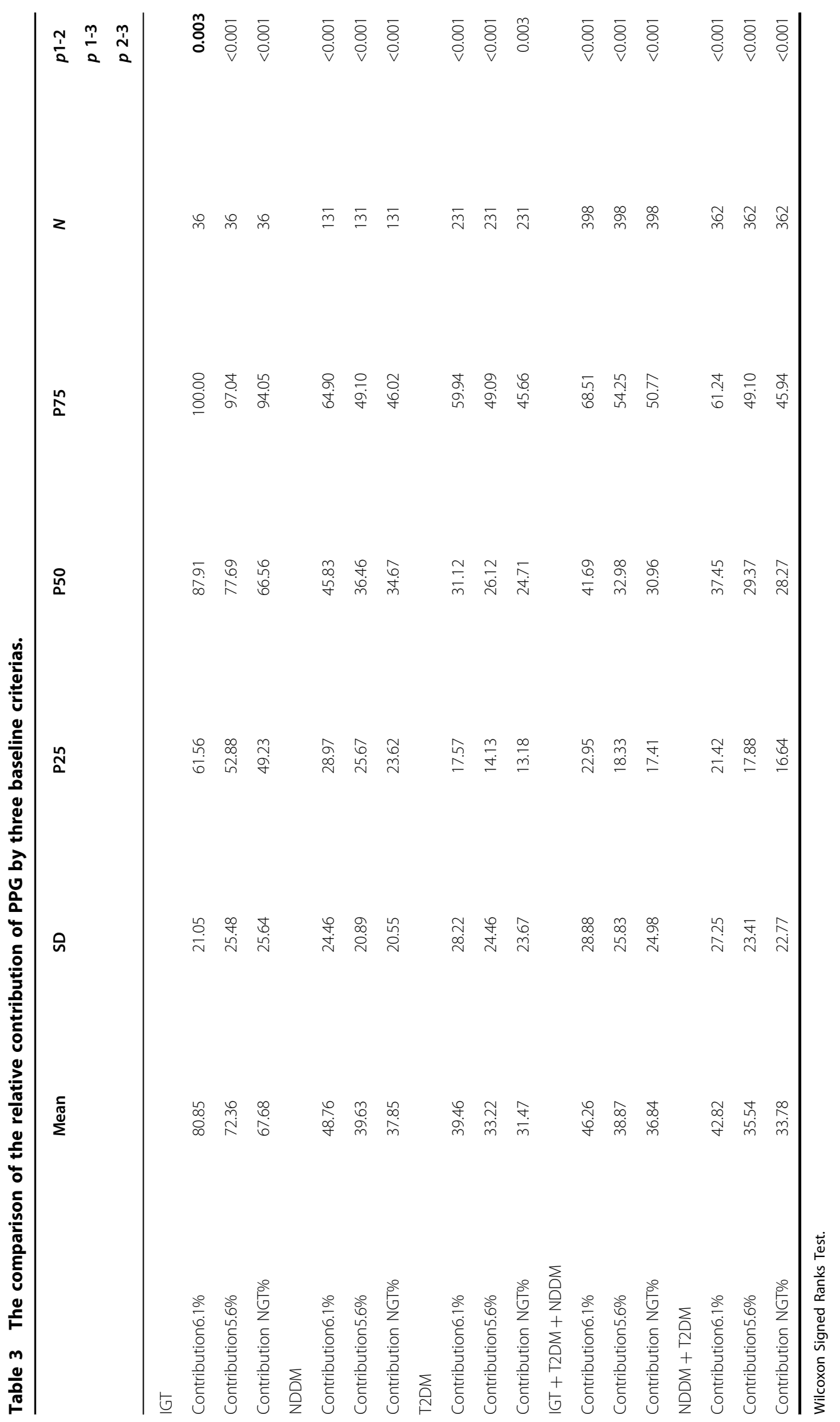




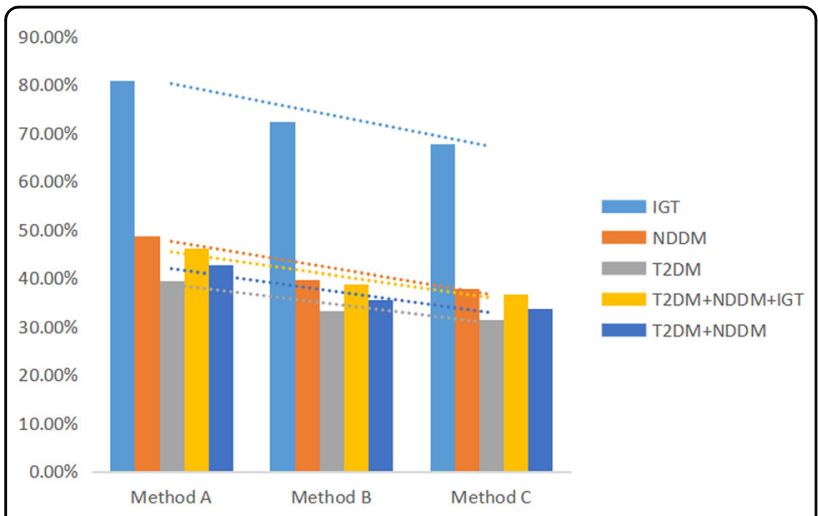

Fig. 3 Relative contribution of PPG to overall hyperglycaemia using three methods in different groups (methods $A, B$, and C used $6.1 \mathrm{mmol} / \mathrm{L}, 5.6 \mathrm{mmol} / \mathrm{L}$, and the $24-\mathrm{h}$ glucose profiles of the NGT curve as baselines, respectively). Different colors represent different groups.

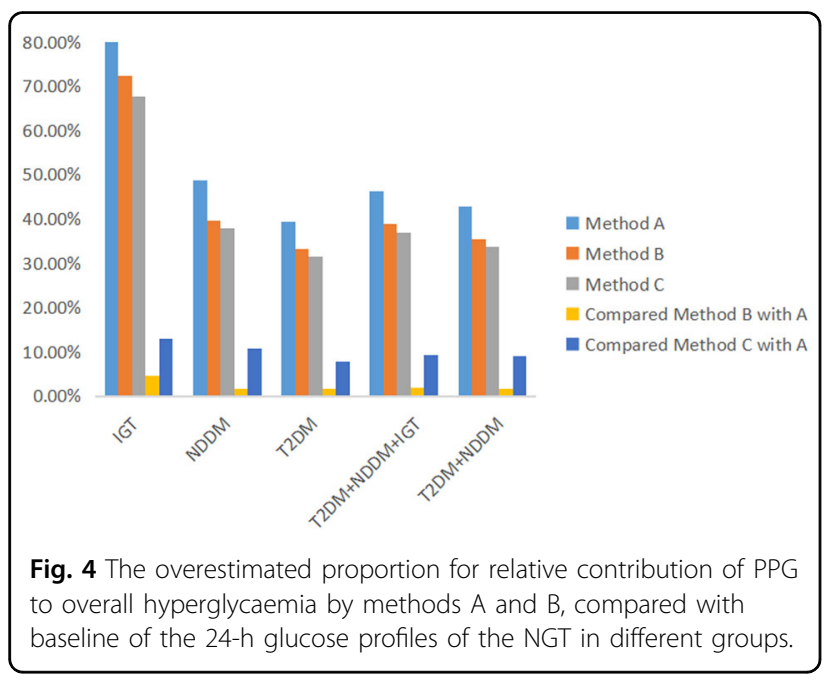

(IGT, NDDM and T2DM) by using CGM and different baseline criteria in a real-world setting (Clinical Trials ID, NCT02648685). In this study, we again verified our previous findings ${ }^{17}$ and found that compared with using the NGT curve as a baseline, the relative contribution of PPG was overestimated by $9.04 \%$ and $1.76 \%$ when 6.1 and $5.6 \mathrm{mmol} / \mathrm{L}$ were used as a baseline, respectively, in subjects from the NDDM and T2DM groups, among all patients with T2DM. However, as the sample size increases, the proportion of this overestimation has shrunk to a certain extent.

Our previous study showed that subjects with NGT exhibited physiological glucose fluctuations after energy intake $^{31}$, and thus, using a fixed value as a baseline to evaluate the BG and PPG contributions is not appropriate because it does not take into account physiological blood glucose fluctuations. Woerle et al. ${ }^{9}$ found that PPG is essential for achieving recommended $\mathrm{HbA1c}$ levels, and most studies have demonstrated that PPG plays an important role in overall hyperglycaemia in patients with good glycaemic control, whereas BG makes a greater contribution to the deterioration of glucose homeostasis. Based on previous studies ${ }^{6,7,13-17}$, we developed a method for quantifying the relative contribution of PPG and BG to hyperglycaemia. A recent meta-analysis ${ }^{32}$ of the correlations of BG and PPG with standard HbA1c showed a pooled correlation coefficient between PPG and HbA1c of $0.68(P<0.001,95 \% \mathrm{CI} ; 0.56-0.75)$, which was slightly higher than that of BG $(r=0.61, P<0.001,95 \% \mathrm{CI}$; 0.48-0.72). The exact contributions of $\mathrm{BG}$ and PPG increments to overall hyperglycaemia remain controversial. The discrepancies between previously published data may have resulted from the interference of several co-variates. First, some studies used the multipoint glucose value or fingerstick glucose sample, whereas others used a CGM for quantification. The former test shows a loss of at least $10 \mathrm{~h}$ of blood glucose data and does not represent integrated glucose fluctuations throughout the day, and FPG cannot reveal the BG levels. Second, the baseline values in these reports were different. Most studies selected $6.1 \mathrm{mmol} / \mathrm{L}$ or $5.6 \mathrm{mmol} / \mathrm{L}$ as the baseline but none used the NGT curve as a baseline. Third, the contribution of PPG may have been overestimated if $\mathrm{AUC}_{-\mathrm{T}}$ was calculated as $\mathrm{AUC}_{\mathrm{T} 2 \mathrm{DM}}-\mathrm{AUC}_{\text {baseline }}$ when the glucose value was under the baseline in some patients with T2DM, leading to more than a fraction of the area under the curve being subtracted. Thus, the area below the baseline point was not included in the calculation of overall hyperglycaemia. In addition, the Asian and Caucasian populations differ in various characteristics such as race and $\operatorname{diet}^{33}$. HbA1c changes slowly and reflects averages over months, whereas CGM reflects actual excursions in minutes. Brown et al. pointed CGM reviewed several clinical scenarios of glycaemic outcomes from CGM data that can be analyzed to describe glycaemic variability and its attendant risks of hyperglycaemia and hypoglycaemia, to enhance interpretation of treatment effect and improve clinical decision-making ${ }^{34}$. In our further research, we will explore the changes of these parameters which from CGM with different levels of HbA1c.

Recent studies of diabetes patients being administered insulin $^{20-22}$ and Glucagon like peptide-1 receptor agonists (GLP-1 RAs) $^{23}$ are a good complement to our research. These studies also revealed that BG plays a major role in the subgroup of patients with HbA1c levels > 8.5\%, and the relative contribution of PPG decreased with increasing HbA1c (from $65.5 \%$ to $39.2 \%$ ). The difference in the relative contribution of PPG between the studies performed by Li et al. ${ }^{20}$ and Riddle's et al. ${ }^{7}$ may be that Riddle 
et al. used $5.6 \mathrm{mmol} / \mathrm{L}$ as a baseline, whereas $\mathrm{Li}$ et al. used $6.1 \mathrm{mmol} / \mathrm{L}$ as a baseline, leading to the overestimation of the relative contribution of PPG. The NGT curve considers physiological fluctuations in normal people after meals, and thus, can be used as a baseline to evaluate the relative contribution of PPG and BG to hyperglycaemia.

A new study on Asian populations conducted by $\mathrm{Moon}^{25}$ found consistent results with us, suggested that BG predominantly contributed to overall hyperglycemia at higher HbA1c levels. Moreover, when controlling for HbA1c and other factors, recommended TG and waist circumference showed a significant correlation with BG in Korean T2DM patients. It has been suggested in previous studies that TG and waist circumference increased fasting glucose by affecting insulin resistance ${ }^{35}$. So, some certain factors should be taken into account when concerning contribution of PPG and prescribing medications for T2DM patients.

This study provides a new approach for investigating the relative contribution of BG and PPG to HbA1c in T2DM by using the NGT curve as a baseline based on comparison of three different baselines. However, this study had some limitations. The survey mainly focused on differences between different baselines in different groups without discussing the differences based on different HbA1c levels. Additionally, this was a single-center study; this may have affected testing efficiency and may not be representative of all persons in China. Further, patients treated with insulin or more complex therapeutic interventions and those experiencing diabetes-related complications excluded from the study, and thus, the conclusions cannot be generalised to a larger population. Prospective research is needed to further understand the impact of various oral anti-diabetes treatment regimens on glycaemic control among patients with T2DM in China.

Our findings have important clinical implications, as the relative contribution changes with the degree of $\mathrm{HbA1c}$, medications should be prescribed accordingly. T2DM is a progressive disease, and the intensity of hyperglycaemia control treatment should be increased accordingly with the development and refinement of $\mathrm{CGM}^{36}$. We know lifestyle intervention should be applied throughout the diabetes treatment process. When diet and exercise cannot effectively control the blood glucose levels, pharmacologic therapy should be provided timely ${ }^{37}$. In prediabetes, the relative contribution of PPG was $67.68 \%$ using the NGT curve as the baseline. That suggests we should focus on controlling the PPG excursion. We could take dietary intervention, or take the pills which control PPG, such as $\alpha$-glucosidase inhibitors and so on. On the contrary, basal hyperglycemia plays a major role as the HbA1c level rises in T2DM. At this time, it is important to make basal hyperglycemia reaching target range. Then, such as basal insulin, metformin, sulfonylurea, DPP-4 inhibitors or Glucagon like peptide-1 receptor agonists
(GLP-1 RAs) should be considered with priority. When the BG decreased, the PPG levels would drop down with the tide. Furthermore, besides considering the level of HbA1c, some other factors should also be considered, such as TG, waist circumference, age, comorbidities and complications. Individualized treatment should be administrated properly and timely, ensuring patients can reach the goals of HbA1c, PPG and BG, at the same time minimize blood sugar fluctuations as soon as possible.

In conclusion, we confirmed that a progressive shift occurs in the respective contributions of BG and PPG with the increasing of glucose, and the contribution of BG is predominant in patients with worsening HbA1c. Moreover, using the NGT curve as a baseline to evaluate the contribution of BG/FG and PPG to HbAlc is accurate and precise, as it considers the physiological fluctuations in normal people after meals. If the NGT curve is not available, the standard of $5.6 \mathrm{mmol} / \mathrm{L}$ can be used, whereas a standard of $6.1 \mathrm{mmol} / \mathrm{L}$ does not appear to be given accurate results.

\section{Acknowledgements}

The authors would like to thank all patients, practice nurses, and investigators for their contributions to this study. We also thank Dr. Yuanyang Zhou for assisting us with this study. This study was supported by the National Natural Science Foundation of China (81471043), Foster Fund of Academic and Technical Leaders in Sichuan Province (JH2014009), Science and Technology Bureau of Sichuan Province (2015SZ0228-4, 2018SZ0239), Funding Projects Sanofi (Beijing) Pharmaceuticals Co., Ltd (H1509084), Science and Technology Bureau of Chengdu city (2017-CY02-00028-GX), National Science and Technology Major Project (2017 ZX09304023), and National Key R\&D Program of China (2017YFC1309605), West China Nursing Discipline Development Special Fund Project, Sichuan University(Grant No. HXHL20005)and 1.3.5 Project for disciplines of excellence, West China Hospital, Sichuan University (Grant No. ZYGD18025).

\section{Author details}

${ }^{1}$ Innovation Center for Wound Repair, Diabetic Foot Care Center, Department of Endocrinology and Metabolism, West China Hospital, Sichuan University, Chengdu, China. ${ }^{2}$ Department of Endocrinology and Metabolism, The First People's Hospital of Longquanyi District, Chengdu, China. ${ }^{3}$ Wannian Community health center in Chenghua district, Chengdu, China. ${ }^{4}$ Shudu Community health center in Xindu district, Chengdu, China. ${ }^{5}$ Chinese Cochrane Centre, Chinese EBM Centre, West China Hospital, Sichuan University, Chengdu, China

\section{Author contributions}

All authors have discussed, read, and approved the final manuscript. M.J. and H.H. conceived and designed the study, collected data, and drafted the manuscript. Y.X., C.D., T.C., Z.L., D.Q., W.X., G.Y. and W.C. designed the study and collected data. L.G. interpreted the data and contributed to the statistical analysis. R.X. designed and coordinated the study, acquired funding, and participated in writing and editing the final manuscript.

\section{Conflict of interest}

The authors declare no competing interests.

\section{Publisher's note}

Springer Nature remains neutral with regard to jurisdictional claims in published maps and institutional affiliations.

Supplementary information The online version contains supplementary material available at https://doi.org/10.1038/s41387-021-00156-1. 
Received: 28 August 2020 Revised: 9 May 2021 Accepted: 18 May 2021 Published online: 04 June 2021

\section{References}

1. Weng, J. et al. Chinese Diabetes Society. Standards of care for type 2 diabetes in China. Diabetes Metab. Res. Rev. 32, 442-458 (2016).

2. Prospective, U. K. Diabetes Study (UKPDS) Group. Intensive blood-glucose control with sulphonylureas or insulin compared with conventional treatment and risk of complications in patients with type 2 diabetes (UKPDS 33). Lancet 352, 837-853 (1998)

3. Monami, M. et al. Fasting and post-prandial glucose and diabetic complication. A meta-analysis. Nutr. Metab. Cardiovasc Dis. 23, 591-598 (2013).

4. Ceriello, A. et al. International Prandial Glucose Regulation Study Group. Postprandial hyperglycaemia and cardiovascular complications of diabetes: an update. Nutr. Metab. Cardiovasc Dis. 16, 453-456 (2006).

5. Mannucci, E., Monami, M., Lamanna, C. \& Adalsteinsson, J. E. Post-prandial glucose and diabetic complications: systematic review of observational studies. Acta Diabetol. 49, 307-314 (2012).

6. Monnier, L., Lapinski, H. \& Colette, C. Contributions of fasting and postprandial plasma glucose increments to the overall diurnal hyperglycemia of type 2 diabetic patients: variations with increasing levels of $\mathrm{HbA}(1 \mathrm{c})$. Diabetes Care. 26, 881-885 (2003).

7. Riddle, M., Umpierrez, G., DiGenio, A., Zhou, R. \& Rosenstock, J. Contributions of basal and postprandial hyperglycemia over a wide range of $\mathrm{A} 1 \mathrm{C}$ levels before and after treatment intensification in type 2 diabetes. Diabetes Care. 34, 2508-2514 (2011)

8. Borg, R. et al. ADAG Study Group. Associations between features of glucose exposure and A1C: the A1C-Derived Average Glucose (ADAG) study. Diabetes 59, 1585-1590 (2010).

9. Woerle, H. J. et al. Impact of fasting and postprandial glycemia on overall glycemic control in type 2 diabetes Importance of postprandial glycemia to achieve target HbA1c levels. Diabetes Res. Clin. Pract. 77, 280-285 (2007).

10. Shimizu, H., Uehara, Y., Okada, S. \& Mori, M. Contribution of fasting and postprandial hyperglycemia to hemoglobin A1c in insulin-treated Japanese diabetic patients. Endocr. J. 55, 753-756 (2008).

11. Peter, R. et al. Relative and absolute contributions of postprandial and fasting plasma glucose to daytime hyperglycaemia and $\mathrm{HbA}(1 \mathrm{c})$ in subjects with type 2 diabetes. Diabet. Med. 26, 974-980 (2009).

12. Kikuchi, K. et al. Correlations of fasting and postprandial blood glucose increments to the overall diurnal hyperglycemic status in type 2 diabetic patients: variations with levels of HbA1c. Endocr. J. 57, 259-266 (2010).

13. Wang, J. S. et al. Contribution of postprandial glucose to excess hyperglycaemia in Asian type 2 diabetic patients using continuous glucose monitoring. Diabetes Metab. Res. Rev. 27, 79-84 (2011).

14. Monnier, L., Colette, C. \& Owens, D. Postprandial and basal glucose in type 2 diabetes: assessment and respective impacts. Diabetes Technol. Ther. 13 S25-S32 (2011)

15. Peter, R., Dunseath, G., Luzio, S. D. \& Owens, D. R. Estimates of the relative and absolute diurnal contributions of fasting and post-prandial plasma glucose over a range of hyperglycaemia in type 2 diabetes. Diabetes Metab. 39, 337-342 (2013)

16. Fysekidis, M. et al. Increased glycemic variability and decrease of the postprandial glucose contribution to $\mathrm{HbA} 1 \mathrm{c}$ in obese subjects across the glycemic continuum from normal glycemia to first time diagnosed diabetes. Metabolism 63, 1553-1561 (2014).
17. Kang, $X$. et al. Contributions of basal glucose and postprandial glucose concentrations to hemoglobin A1c in the newly diagnosed patients with type 2 diabetes-the preliminary study. Diabetes Technol. Ther. 17, 445-448 (2015).

18. Wang, X., Wang, F., Wang, H. \& Li, N. Correlation of fasting versus postprandial plasma glucose with $\mathrm{HbA1C}$ in Chinese type 2 diabetic patients taking different hypoglycemic agents. Clin. Lab. 63, 1293-1300 (2017).

19. Lim, L. L. et al. Relationship of glycated hemoglobin, and fasting and postprandial hyperglycemia in type 2 diabetes mellitus patients in Malaysia. J. Diabetes Investig. 8, 453-461 (2017).

20. Li, W. et al. Contribution of BHG and PPHG to overall hyperglycemia in T2DM patients treated with LM25 and LM50: post hoc analysis of a randomized crossover trial. Diabetes Ther. 9, 1605-1614 (2018).

21. Reznik, Y., Habteab, A., Castaneda, J., Shin, J. \& Joubert, M. Contribution of basal and postprandial hyperglycaemia in type 2 diabetes patients treated by an intensified insulin regimen: Impact of pump therapy in the OPT2mise trial. Diabetes Obes. Metab. 20, 2435-2441 (2018).

22. Su, Q., Liu, J., Li, P., Qian, L. \& Yang, W. Relative contribution of fasting and postprandial blood glucose in overall glycemic control: post hoc analysis of a phase IV randomized trial. Diabetes Ther. 9, 987-999 (2018).

23. Umpierrez, G. et al. Relative contribution of basal and postprandial hyperglycaemia stratified by $\mathrm{HbA} 1 \mathrm{c}$ categories before and after treatment intensification with dulaglutide. Diabetes Obes. Metab. 21, 1365-1372 (2019).

24. Yan, R. et al. Contributions of fasting and postprandial glucose concentrations to haemoglobin A1c in drug-native Mal-glucose metabolism in Chinese population using continuous glucose monitoring system. Int J. Endocrinol. 1, 1267475 (2019). 2019.

25. Moon, J. et al. Fasting and postprandial hyperglycemia: their predictors and contributions to overall hyperglycemia in Korean patients with type 2 diabetes. Endocrinol. Metab. 35, 290-297 (2020).

26. American Diabetes Association. Classification and diagnosis of diabetes. Dia betes Care. 38, S8-S16 (2015). (2)Suppl.

27. Alberti, K G. \& Zimmet, P. Z. Definition, diagnosis and classification of diabetes mellitus and its complications. Part 1: diagnosis and classification of diabetes mellitus provisional report of a WHO consultation. Diabet. Med. 15, 539-553 (1998).

28. Bergenstal, R. M. Continuous glucose monitoring: transforming diabetes management step by step. Lancet 391, 1334-1336 (2018).

29. Li, W. et al. Effects of insulin lispro mix 25 and insulin lispro mix 50 on postprandial glucose excursion in patients with type 2 diabetes: a prospective, open-label, randomized clinical trial. Diabetes Ther. 9, 699-711 (2018).

30. Chinese Diabetes Society. China Medical Nutritional Therapy Guideline for Diabetes (2013). Diabetes World 10, 289-307 (2013).

31. Kang, $X$. et al. Effects of different proportion of carbohydrate in breakfast on postprandial glucose excursion in normal glucose tolerance and impaired glucose regulation subjects. Diabetes Technol. Ther. 15, 569-574 (2013).

32. Ketema, E. B. \& Kibret, K. T. Correlation of fasting and postprandial plasma glucose with $\mathrm{HbA1c}$ in assessing glycemic control; systematic review and meta-analysis. Arch. Public Health 73, 43-46 (2015).

33. Wolffenbuttel, B. H. et al. Ethnic differences in glycemic markers in patients with type 2 diabetes. Diabetes Care. 36, 2931-2936 (2013).

34. Brown, S. A. et al. Beyond HbA1c: using continuous glucose monitoring metrics to enhance interpretation of treatment effect and improve clinical decision-making. Diabet. Med. 36, 679-687 (2019).

35. Lin, S. X. et al. Does elevated plasma triglyceride level independently predict impaired fasting glucose? the Multi-Ethnic Study of Atherosclerosis (MESA) Diabetes Care. 36, 342-347 (2013).

36. Robert, A. V. Going beyond $\mathrm{HbA1c}$ to understand the benefits of advanced diabetes therapies. J. Diabetes 11, 23-31 (2019).

37. Jia, W. et al. On behalf of Chinese Diabetes Society. Standards of medical care for type 2 diabetes in China 2019. Diabetes Metab. Res. Rev. 35, 3158 (2019). 